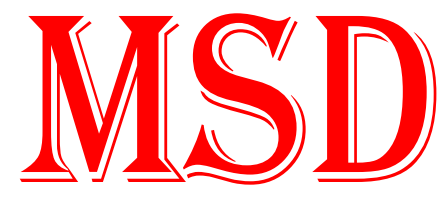

Medical Science and Discovery ISSN: $2148-6832$

\title{
Speech characteristics of parkinson disease
}

\author{
Zeynep Zeliha Sonkaya ${ }^{1}$, Mustafa Ceylan ${ }^{2}$, Ali Riza Sonkaya ${ }^{3}$ \\ 1 Ankara University, Department of Linguistics and Literature, Ankara, TR \\ 2 Atatürk University, Department of Neurology, Erzurum, TR \\ 3 University of Health Science, Gülhane School of Medicine, Department of Neurology, Ankara, TR \\ * Corresponding Author: Zeynep Zeliha Sonkaya E-mail: drzeynepsonkaya@ gmail.com
}

\begin{abstract}
Objective: Parkinson Disease (PD) is known the second most frequent neurodegenerative age-related disorder after Alzheimer's disease. Although over the six million people worldwide suffer from $\mathrm{PD}$, the main cause of the disease remains are unknown. Speech and language impairments have emerged in most patients with PD during the course of the disease. However, clinical profiles or characteristics that might differentiate individuals with PD who are predisposed to speech and language deficits are generally overlooked. Moreover, factors that expedite language disability have still been remained elusive. It is thought that the awareness of speech and language impairments in PD can significantly help to maintain language abilities as the disease progresses and also may contribute to improving communication skills with patients. For this reason, the present study aims to constitute a comprehensive frame for the speech and language characteristics of individuals with PD.
\end{abstract}

Keywords: Parkinson disease, language impairments, speech characteristics

\section{INTRODUCTION}

PD is one of the most common neurodegenerative chronic disorder characterized by progressive loss of dopaminergic neurons, primarily in the substantia nigra pars compacta (1). The disease is particularly featured by motor symptoms such as bradykinesia, rigidity, resting tremor, postural instability, gait problems, and non-motor symptoms like sleep problems, anxiety, and cognitive impairments (2).

PD diagnosis is generally performed according to the certain criteria which depends on the clinical symptom assessment, but these clinical symptoms do not occur until loss of dopaminergic neurons reaches a level of $60-80 \%$ [3,4]. Therefore, in some cases clinical evaluation may be inadequate or lead to misdiagnosis, in particular, at the onset of the disease. On the other hand, there is no exact procedure for a definitive diagnosis of PD. However, in recent studies, it has been shown that speech can be used as an early marker for identification of PD (2).

In the literature, it was determined some important speech manifestations showing the early development of PD which can be summarized as vocal changes, poor articulation, trembling or hoarseness, frequency changes, degraded sound quality, lower tone, decreased rhythm and tonal changes (3-5). Although these manifestations are considered as one of the cardinal symptoms of PD, there is a limited number of studies about speech characteristics of the disease. Therefore, the present study aims to draw a comprehensive frame for the speech characteristics of individuals with PD.

\section{Speech Features in PD}

Speech is a complex mechanism, and it arises from sequential or synchronous integration of the central nerve system. The integration of signals sent during the speech is provided with neuromuscular coordination of peripheral level of brain functions. Damage to the motor speech areas of the brain results a number of speech disorders. Diagnostic features of patients with neurological dysfunction are generally seen in the form of difficulty in word finding, impaired speech, prolonged speech time or pronunciation ambiguities. By using these characteristic features, practical research can be done to the patients performing via neurological applications and also it can be investigated the relationship between protatic structure and speech deformation. 
In this manner, coordination anomalies in the neuromuscular system are reflected in the patients' speech in the form of nasal emission, the spread of sound quality to the nasal cavity, the neglect of the voice, the skipping of the voice, the substitution of another voice and vocal disorders (6).

As it is well known speech impairments are quite common in patients with PD. Especially, spontaneous speech is severely reduced. Also, "making verbal communication slower and less accurate, and deficits in verb inflection, verbal fluency, and verb generation" are often seen in this disease (1). As a matter of fact, the prevalence of speech impairments among patients with PD have been reported to be as high as $89 \%$ (4). In one of the oldest epidemiology studies made by Atarachi and Uchida (1959), it was found to be speech disturbances in more than two-thirds of individuals suffering from PD (7). Likewise, nowadays as well, it is stated that approximately half of all patients with PD display some kind of speech impairments (8). In particular, dysarthria which is one of the types of speech impairments comes into prominence, because it is accepted as a universal data in PD (3). However, it is stated in the literature more than $90 \%$ of patients with PD suffer from dysarthria (5).

In the earliest studies about dysarthria, a number of researchers tried to make a rather wide-ranging neurological classification of this speech impairment. First of all, Zentay (1937) suggested three subdivisions based on neuroanatomical involvement consisting of "fronto-pontocerebellar, cortico-striato-pallido-rubro-bulbar-extrapyramidal and cortico-bulbar areas" (9). Another researcher Foreschel (1943) offered a different classification which comprised of "pyramidal, extrapyramidal, frontopontine and cerebellar distinctions" (10). Also, Brain (1948) made a classification that encompassed "upper motor neurons, corpus striatum, and lower motor neuron lesions, myopathies, and disorders of coordination" (9). Drawing on previous classifications of dysarthrias, in one study which was aimed to define speech impairments in populations with subcortical damage, Peacher (1950) attempted to establish a relationship between features of dysarthria and central and peripheral nervous system lesions. As a result of the study, the author reported that "pure dysarthria", which means misarticulation alone, seldom takes place with central nervous system lesions, but is more often encountered after peripheral nervous system lesions (10).

The acoustics of dysarthric speech reflect the anatomical and physiological changes caused by PD (11). At first, the emerged changes in the respiratory system influence the vocal intensity of the individuals with PD (12). As a second, the emerged changes in the phonatory system mainly affects the vibratory rhythm of the vocal folds, which causes the fundamental frequency increase and prosody alteration (11). Finally, the articulatory system is influenced as well, causing in articulatory inaccuracies and an increase in the number of pauses (13). Although changes in voice quality and intensity are accepted as common early symptoms of dysarthric speech, and this can be detected in the pre-diagnosis process (14), the articulation changes are more likely to emerge in the later stage of the stage (15). On the other hand, a majority number of the patients with PD are unaware that their voice is weak, nor that the voice progressively weakens unless they make a continuous effort during speech (11). In the literature, depending on the location of neurological damage, different types of dysarthria have been classified such as flaccid dysarthria, spastic dysarthria, ataxic dysarthria, hypokinetic dysarthria, hyperkinetic dysarthria, unilateral upper motor neuron dysarthria and mixed dysarthria (3).

Among the types of dysarthria, hypokinetic dysarthria, defined as a slow, weak and monotonous speech, involving motor dysfunctions in respiration, articulation, phonation, resonance, and prosody $(11,16)$, and characterized by "reduced vocal loudness, monotone, reduced fundamental frequency range, consonant and vowel imprecision, breathiness and irregular pauses" is observed quite often in PD $(17,18)$. As a matter of fact, it has been reported nearly $90 \%$ of patients with PD have hypokinetic dysarthria (19). In one study made by Fabbri et al. (2017) and conducted on 27 patients with late-stage PD, it was found to be $89 \%$ of patients with $\mathrm{PD}$ have tendency to produce hypokinetic dysarthria as characterized by "imprecise articulation, prosody abnormalities, disturbance of speech rate, and low vocal volume" (20). On the other hand, Whitfield et al. (2017) demonstrated the impaired coordination of speech production in hypokinetic dysarthria is parallel to impaired motor planning in PD such as muscle rigidity, bradykinesia and tremor (21). However, a great number of neuroimaging research have focused on the mechanism of hypokinetic dysarthria in PD. In one fMRI study carried out by Arnold et al. (2014) it was found to be significant correlation between hypokinetic dysarthria and pathomechanism of PD. As a result of the study, authors reported that hypokinetic dysarthria originates from dysfunction in the basal ganglia motor loop which causes deficiencies in the regulation of amplitude, initiation and velocity of movement (22). Likewise, in a PET study Pinto et al. (2004) showed that hypokinetic dysarthria in patients with PD is associated with functional anomalies in the "basal ganglia, orofacial motor cortex, and cerebellum, together with an increased recruitment of premotor and prefrontal cortices during speech production" (23).

\section{Language Production in PD}

Language production is formed with a combination of many processes included basic language functions and general cognitive functions, as well. The etiology of language impairments in PD is not definitive; however, a great number of studies have focused on an association with handicapped cognition (1). Besides, while in some studies it was hypothesized that language skills in individuals suffer from PD correlate with cognitive abilities $(24,25)$, in other studies more specifically it was connected language impairments to working memory and executive function (19). Furthermore, clinical observations have recommended that language performance in patients with PD can be affected from some degree of depression. In one early study conducted by Starkstein et al. (1989), it was reported that PD patients with major depression performed significantly worse than nondepressed PD patients on all parts of neuropsychological function tests, comprising language tasks (26). However, in another study made by Costa et al. (2006), it was not found to be differences in language function among PD patients with minor depression or major depression and the controls (27). As a result, to our knowledge, there is no consensus on how depression affects the language functions. Although language 
impairments are seen common in PD, not all patients are affected, even over the long-term progression of the disease (28). It is pointed out in the literature that "clinical profiles or features that might differentiate patients who are predisposed to language impairment have been generally overlooked, and factors that precipitate language disability still remain elusive" (1).

Studies about language production in PD are relatively rare. However, multidisciplineer studies have been carried out and various methodologies are employed in these studies to evaluate the language production skills in PD from different perspectives. In one study conducted by Illes et al (1988) aimed to examine language production in PD in terms of acoustic and linguistic perspective. In the study, speech rate, fluency, syntactic complexity, lexical production, and the relative distribution of content and grammatical phrases were evaluated in 10 patients with PD and 10 age-matched controls while reading the "Grandfather" passage and producing spontaneous speech. As a result of the study, authors stated that the language production of patients with PD differed both acoustically and linguistically from healthy older adults. Also, they demonstrated the speech disfluency in PD group. However, authors reported to be significant correlation between syntactic complexity and PD severity, as evaluated by the Webster scale (29). In another study, Murray and Lenz (2001) investigated the language production in conversational discourse of 10 persons with PD, 9 with Huntington's disease, and 17 controls. As a result of the study authors reported no impairments in syntax among those with PD. However, they remarked the significant positive relationships between degree of dementia, length of utterances and sentence complexity in the PD group (30).

Verbal fluency deficits have also been studied in PD. In one meta-analysis study Henry and Crawford (2004) stated to be significant impairment on measures of both semantic and phonetic fluency in patients with PD. In addition, authors reported the semantic fluency deficit seems to be significantly larger than the phonetic deficit and independent of cognitive speed and effortful retrieval, pointed out that semantic memory is particularly impaired in PD (31). On the other hand, in an interesting study examining spontaneous speech in bilingual individuals with PD conducted by Zanini et al. (2010), it was found that patients with PD exhibited more grammatical errors than the controls, only for their first and not their second language (24).

As a consequence of the study, authors suggested that "first language is more likely to reflect implicit, procedural processing and hence more likely to engage basal ganglia structures, which are impaired with PD. In contrast, a participant's second language is more likely to reflect explicit processing and hence more likely to engage neocortical structures" (24). More recently, in a fMRI study made by Coleman et al. (2009), it was reported a more specific grammatical impairment in PD, a deficit in terms of producing a correct verb form, whereas noun production was not impaired (32).

Prosodic abnormalities are often recognized to be present in the speech of individuals with PD (33). In one study, Pell et al. (2006) pointed out that prosodic disturbances appear early in the disease course of PD and may be present prior to diagnosis in some cases (34). In another study, Kent and
Rosenbek (1982) investigated the acoustic speech features of patients with prosodic disturbances due to a variety of central nervous system lesions. As a result of the study, authors summarized the acoustic findings associated with reduced prosody in PD as reflecting an overall "reduction of acoustic contrast or detail" (35). Also, Pell and Leonard (2005) examined the ability of older adults with and without PD to recognize emotions from prosody, facial expressions, and verbal cues. In the study, patients with PD were found to be significantly impaired in their ability to notice emotions based exclusively on prosody (36).

However, authors reported that patients' ability to specify emotions in other modalities (i.e., facial expression, verbal cues) was comparatively preserved, suggesting that prosodic decoding of emotions seems to be differentially influenced in PD. Interestingly, specifying the emotion of disgust from prosody was particularly impaired in individuals with PD (36). Lloyd (1999) also studied prosodic perception features of patients with PD. Findings demonstrated that nondemented PD patients exhibited the impaired ability to accurately identify utterance prosody and displayed deficits for utterance prosody comprehension. As a result of the study, author suggest that verbal and emotional nuances, such as a subtle change in tone of voice or facial expression, may go undetected by patients with PD (37).

Pragmatic abilities, which refers to the social language skills that people use in their daily interactions with others, are impaired in PD. Despite reported evidence, the neuropsychological picture of the pragmatic profile of PD is still unclear (38). Impairment of pragmatic abilities in PD has been for long studied in relation with attentional, short-term memory, and executive deficits (39).

An early report investigating pragmatic production deficits in PD was conducted by McNamara and Durso (2003), in which, patients with PD and control group engaged in brief conversations with a member of the research team. In the study, conversations were coded using the pragmatic checklist. Scheme for classifying social language skills were determined in terms of verbal features such as topic selection, topic maintenance, lexical variation, paralinguistic features such as prosody, vocal quality, and nonverbal features such as gaze, and gestures. As a result of the study, it was reported that patients with PD were impaired on $20.4 \%$ of the items relative to the controls. In addtition, both patients with PD and controls did not vary significantly on measures of mental status or verbal fluency. Authors commented these findings that the pragmatic disturbance was not simply reducible to global cognitive deficits or poverty of speech (40). In another study, it was demonstrated the specific pragmatic difficulties in patients with PD, in terms of reduced spontaneous speech production, poor conversational appropriateness, prosody impairment or slowness in processing speed (29). However, there is still lacks a comprehensive description of the pragmatic profile characteristic of $\mathrm{PD}$ in the literature. Furthermore, some important aspects that could be associated with pragmatic abilities in PD have been neglected. As a result of this, it can be said, from a clinical perspective, little is known about factors that might help to maintain pragmatic abilities in patients with PD as the disease progresses. 


\section{CONCLUSION}

Most patients with PD develop speech and language impairments during the course of the disease. In the literature, it was determined some important speech and language manifestations showing the early development of PD. However, clinical profiles or features that might differentiate patients who are predisposed to speech and language deficits are generally overlooked, moreover, factors that precipitate language disability still remain elusive. It is thought that awareness of these speech and language deficits in PD can greatly help to maintain language abilities as the disease progresses and also may contribute to improve communication skills with patients. Therefore, multidisciplineer studies such as collaboration of neurology, software engineering and linguistics are required to diagnosis and treatment of speech and language disorders.

\section{Acknowledgement: None}

Author Contributions: ZZS, MC, ARS: Study design, Concept, Data collection and/or processing, Analysis and/or interpretation, Literature review, ZZS, ARS: Writing, Revisions

Conflict of interest: The author declared no potential conflicts of interest with respect to the research, authorship, and/or publication of this article. This research did not receive and specific grant from funding agencies in the public, commercial, or not-for-profit sectors.

Ethical approval: The study was conducted according to the guidelines of the Declaration of Helsinki and approved by Local Ethical Committee.

\section{REFERENCES}

1. Liu L, Luo X-G, Dy C-L, Ren Y, Feng Y, Yu H-M, et al Characteristics of language impairment in Parkinson's disease and its influencing factors. Translational Neurodegeneration. 2015;4(1):1-8.

2. Sonkaya AR, Ceylan M. The investigation of alexithymia in patients with Parkinson disease. Gülhane Tip Dergisi. 2019;61(3):93.

3. Yang S, Wang F, Yang L, Xu F, Luo M, Chen X, et al. The physical significance of acoustic parameters and its clinical significance of dysarthria in parkinson's disease. Scientific Reports. 2020;10(1):1-9.

4. Dashtipour K, Tafreshi A, Lee J, Crawley B. Speech disorders in Parkinson's disease: pathophysiology, medical management and surgical approaches. Neurodegenerative disease management. 2018;8(5):337-48

5. Muñoz-Vigueras N, Prados-Román E, Valenza MC, Granados-Santiago M, Cabrera-Martos I, Rodríguez-Torres J, et al. Speech and language therapy treatment on hypokinetic dysarthria in Parkinson disease: Systematic review and meta-analysis. Clinical Rehabilitation. 2020:0269215520976267.

6. Bayazıt ZZ, Sonkaya AR. Multipl Skleroz Erken Tanısında Ses Analizi Yönteminin Kullanılabilirliğinin İncelenmesi. Online Türk Sağlık Bilimleri Dergisi. 2019;4(4):413-27.

7. Atarachi J, Uchida E. A clinical study of Parkinsonism. Re cent Adv Res Nerv Syst 1959; 3: 871. 1959;882.

8. Zarifkar P, Kim J, La C, Zhang K, YorkWilliams S, Levine TF, et al. Cognitive impairment in Parkinson's disease is associated with Default Mode Network subsystem connectivity and cerebrospinal fluid A $\beta$. Parkinsonism \& Related Disorders. 2021;83:71-8.
9. Cohen H. Disorders of speech and language in Parkinson's disease. Mental and behavioral dysfunction in movement disorders: Springer; 2003. p. 125-34.

10. Peacher WG. The etiology and differential diagnosis of dysarthria Journal of Speech and Hearing Disorders. 1950;15(3):252-65.

11. Fernández-García S, Dumitrache CG, González-López JA. Acoustic analysis of the voice in patients with Parkinson's disease and hypokinetic dysarthria. Revista de Logopedia, Foniatría y Audiología. 2020 .

12. Watts CR. A retrospective study of long-term treatment outcomes for reduced vocal intensity in hypokinetic dysarthria. BMC Ear, Nose and Throat Disorders. 2016;16(1):1-7.

13. Sveinbjornsdottir S. The clinical symptoms of Parkinson's disease Journal of neurochemistry. 2016;139:318-24

14. Oguz H, Tunc T, Safak MA, Inan L, Kargin S, Demirci M. Objective voice changes in nondysphonic Parkinson's disease patients. Journal of otolaryngology. 2006;35(5).

15. Wight S, Miller N. Lee Silverman Voice Treatment for people with Parkinson's: audit of outcomes in a routine clinic. International journal of language \& communication disorders. 2015;50(2):215-25.

16. Duffy JR. Motor Speech disorders-E-Book: Substrates, differential diagnosis, and management: Elsevier Health Sciences; 2013.

17. Levy ES, Moya-Galé G, Chang YHM, Freeman K, Forrest K, Brin MF, et al. The effects of intensive speech treatment on intelligibility in Parkinson's disease: a randomised controlled trial. EClinicalMedicine. 2020;24:100429.

18. Moya-Galé G, Levy ES. Parkinson's disease-associated dysarthria: prevalence, impact and management strategies. Research and Reviews in Parkinsonism. 2019;9:9-16.

19. Altmann LJ, Troche MS. High-level language production in Parkinson's disease: a review. Parkinson's Disease. 2011;2011.

20. Fabbri M, Guimarães I, Cardoso R, Coelho M, Guedes LC, Rosa MM et al. Speech and voice response to a levodopa challenge in late-stage Parkinson's disease. Frontiers in neurology. 2017;8:432.

21. Whitfield JA, Goberman AM. Speech motor sequence learning: acquisition and retention in Parkinson disease and normal aging. Journal of Speech, Language, and Hearing Research. 2017;60(6):147792.

22. Arnold C, Gehrig J, Gispert S, Seifried C, Kell CA. Pathomechanisms and compensatory efforts related to Parkinsonian speech. NeuroImage: Clinical. 2014;4:82-97.

23. Pinto S, Thobois S, Costes N, Le Bars D, Benabid AL, Broussolle E, et al. Subthalamic nucleus stimulation and dysarthria in Parkinson's disease: a PET study. Brain. 2004;127(3):602-15.

24. Zanini S, Tavano A, Fabbro F. Spontaneous language production in bilingual Parkinson's disease patients: Evidence of greater phonological, morphological and syntactic impairments in native language. Brain and language. 2010;113(2):84-9.

25. Colman KS, Koerts J, van Beilen M, Leenders KL, Post WJ, Bastiaanse $\mathrm{R}$. The impact of executive functions on verb production in patients with Parkinson's disease. cortex. 2009;45(8):930-42.

26. Starkstein SE, Preziosi TJ, Berthier ML, Bolduc PL, Mayberg HS Robinson RG. Depression and cognitive impairment in Parkinson's disease. Brain 1989:112(5):1141-53.

27. Costa A, Peppe A, Carlesimo G, Pasqualetti P, Caltagirone C. Major and minor depression in Parkinson's disease: a neuropsychological investigation. European Journal of Neurology. 2006;13(9):972-80. 
28. Hartelius L, Svensson P. Speech and swallowing symptoms associated with Parkinson's disease and multiple sclerosis: a survey. Folia phoniatrica et logopaedica. 1994;46(1):9-17.

29. Illes J, Metter E, Hanson W, Iritani S. Language production in Parkinson's disease: Acoustic and linguistic considerations. Brain and language. 1988;33(1):146-60.

30. Murray LL, Lenz LP. Productive syntax abilities in Huntington's and Parkinson's diseases. Brain and Cognition. 2001;46(1-2):213-9.

31. Henry JD, Crawford JR. Verbal fluency deficits in Parkinson's disease: a meta-analysis. Journal of the International Neuropsychologica Society: JINS. 2004;10(4):608.

32. Coleman MR, Davis MH, Rodd JM, Robson T, Ali A, Owen AM, et al. Towards the routine use of brain imaging to aid the clinical diagnosis of disorders of consciousness. Brain. 2009;132(9):2541-52.

33. Jones HN. Prosody in Parkinson's disease. Perspectives on Neurophysiology and Neurogenic Speech and Language Disorders. 2009;19(3):77-82.
34. Pell MD, Cheang HS, Leonard CL. The impact of Parkinson's disease on vocal-prosodic communication from the perspective of listeners. Brain and language. 2006;97(2):123-34.

35. Kent RD, Rosenbek JC. Prosodic disturbance and neurologic lesion. Brain and language. 1982;15(2):259-91.

36. Pell MD, Leonard CL. Facial expression decoding in early Parkinson's disease. Cognitive Brain Research. 2005;23(2-3):327-40.

37. Lloyd A. Comprehension of prosody in Parkinson's disease. Cortex. 1999;35(3):389-402.

38. Holtgraves T, Cadle C. Communication impairment in patients with Parkinson's disease: Challenges and solutions. Research and Reviews in Parkinsonism. 2016;6:45-55

39. Montemurro S, Mondini S, Signorini M, Marchetto A, Bambini V, Arcara G. Pragmatic language disorder in Parkinson's disease and the potential effect of cognitive reserve. Frontiers in psychology. 2019;10:1220.

40. McNamara P, Durso R. Pragmatic communication skills in patients with Parkinson's disease. Brain and language. 2003;84(3):414-23.

Copyright (C) 2021 The Author(s); This is an open-access article distributed under the terms of the Creative Commons Attribution License (http://creativecommons.org/licenses/by/4.0), (CC BY NC) which permits unrestricted use, distribution, and reproduction in any medium, provided the original work is properly cited. International Journal of Medical Science and Discovery. 